S1

Supporting information for

\title{
Synthesis and Reactivity of Mixed Alkynylalanes by Direct Triethylamine-Catalyzed
} Alumination of Terminal Alkynes.

Christophe Feuvrie, Jérôme Blanchet, Martine Bonin* and Laurent Micouin.*

Laboratoire de Chimie Thérapeutique, UMR 8638 associée au CNRS et à l'Université René

Descartes, Faculté des Sciences Pharmaceutiques et Biologiques, 4 av. de l'Observatoire, 75270 Paris cedex 06 (France)

Compounds 6a, $\mathbf{6 b}$ and 7a-e have already been reported elsewhere:

6a, 6b : Blanchet, J.; Bonin, M.; Micouin, L.; Husson, H.-P. J. Org. Chem, 2000, 65, 6423

7a, 7d : Ahn, J. H.; Joung, M. J.; Yoon, N. M., J. Org. Chem, 1995, 60, 6173

7b : Yamaguchi, M.; Hayashi, A.; Minami, T. J. Org. Chem, 1991, 56, 4091

7c : Uma, R.; Davies, M. K.; Crévisy, C.; Grée, R. Eur. J. Org. Chem. 2001, 3141

7e : Liang, C. K. Bull. Soc. Chim. Fr. 1933, 53, 33.

2-[ferrocenylmethyl-(6-chloro-1-phenethyl-hex-2-ynyl)-amino]-2-phenylethanol 6c oil, $[\alpha]_{\mathrm{D}}=+20\left(\mathrm{c}=1.00, \mathrm{CHCl}_{3}\right) ;{ }^{1} \mathrm{H} \mathrm{NMR}\left(400 \mathrm{MHz}, \mathrm{CDCl}_{3}\right) \delta 1.71(\mathrm{q}, J=7.6 \mathrm{~Hz}, 2 \mathrm{H}), 1.94$ (quint, $J=6.4 \mathrm{~Hz}, 2 \mathrm{H}), 2.43(\mathrm{t}, J=6.4 \mathrm{~Hz}, 2 \mathrm{H}), 2.50(\mathrm{t}, J=7.9 \mathrm{~Hz}, 2 \mathrm{H}), 3.61(\mathrm{~m}, 4 \mathrm{H}), 3.76-$ $3.87(\mathrm{~m}, 2 \mathrm{H}), 4.02-4.16(\mathrm{~m}, 11 \mathrm{H}), 7.00(\mathrm{~d}, J=7.4 \mathrm{~Hz}, 2 \mathrm{H}), 7.14-7.36(\mathrm{~m}, 8 \mathrm{H}) ;{ }^{13} \mathrm{C} \mathrm{NMR}$ $\left(75 \mathrm{MHz}, \mathrm{CDCl}_{3}\right) \delta 16.3,31.5,32.9,36.7,43.8,45.8,51.9,61.7,65.4,68.0,68.7,70.0,81.4$ 83.8, 86.3, 125.9, 127.6, 128.4, 128.5, 128.8, 139.4, 141.8; IR $\left(\mathrm{cm}^{-1}\right) 3513,3072,2359,1679$, 1494; MS (CI) $555\left(\mathrm{MH}^{+}\right)$.

6-Hept-1-ynyl-1-(2-hydroxy-1-phenyl-ethyl)-piperidine-2-carbonitrile 9a oil, $[\alpha]_{\mathrm{D}}=-87$ $\left(\mathrm{c}=0.96, \mathrm{CH}_{2} \mathrm{Cl}_{2}\right) ;{ }^{1} \mathrm{H} \mathrm{NMR}\left(400 \mathrm{MHz}, \mathrm{CDCl}_{3}\right) \delta 0.93(\mathrm{t}, J=7.2 \mathrm{~Hz}, 3 \mathrm{H}), 1.33-1.51(\mathrm{~m}$, 
5H), $1.57-1.68(\mathrm{~m}, 4 \mathrm{H}), 1.83-1.86(\mathrm{~m}, 2 \mathrm{H}), 1.93-2.00(\mathrm{~m}, 2 \mathrm{H}), 2.29(\mathrm{td}, J=7.1,2.1 \mathrm{~Hz}$, 1H), $3.57(\mathrm{dd}, J=4.9,2.1 \mathrm{~Hz}, 1 \mathrm{H}), 3.88(\mathrm{ddd}, J=11.0,6.9,3.4 \mathrm{~Hz}, 1 \mathrm{H}), 3.99(\mathrm{dd}, J=5.0,3.4$ $\mathrm{Hz}, 1 \mathrm{H}), 4.07(\mathrm{dt}, J=11.3,5.6 \mathrm{~Hz}, 1 \mathrm{H}), 4.17(\mathrm{~m}, 1 \mathrm{H}), 7.25-7.50(\mathrm{~m}, 5 \mathrm{H}) ;{ }^{13} \mathrm{C} \mathrm{NMR}(75$ $\left.\mathrm{MHz}, \mathrm{CDCl}_{3}\right) \delta 14.2,17.0,18.9,22.3,28.2,29.2,31.3,46.1,47.4,63.9,67.0,75.8,89.3$, 118.4, 128.4, 128.5, 129.2, 139.2 ; IR $\left(\mathrm{cm}^{-1}\right)$ 3478, 2931, 2360, 2342, 1622, 1492, 1254 ; HRMS for $\mathrm{C}_{21} \mathrm{H}_{28} \mathrm{~N}_{2} \mathrm{ONa}$ calcd. 347.2099; found 347.2097.

6-(5-Chloro-pent-1-ynyl)-1-(2-hydroxy-1-phenyl-ethyl)-piperidine-2-carbonitrile 9b oil, $[\alpha]_{\mathrm{D}}=-85\left(\mathrm{c}=0.87, \mathrm{CH}_{2} \mathrm{Cl}_{2}\right) ;{ }^{1} \mathrm{H}$ NMR $\left(400 \mathrm{MHz}, \mathrm{CDCl}_{3}\right) \delta 1.53(\mathrm{t}, J=5.9 \mathrm{~Hz}, 1 \mathrm{H}), 1.70$ (m, 2H), 1.80- $2.09(\mathrm{~m}, 6 \mathrm{H}), 2.50(\mathrm{td}, J=6.5,1.4 \mathrm{~Hz}, 2 \mathrm{H}), 3.57(\mathrm{~m}, 1 \mathrm{H}), 3.73(\mathrm{t}, J=6.4,2 \mathrm{H})$, $3.86(\mathrm{~m}, 1 \mathrm{H}), 3.96(\mathrm{~m}, 1 \mathrm{H}), 4.05(\mathrm{dt}, J=11.2,5.0 \mathrm{~Hz}, 1 \mathrm{H}), 4.18(\mathrm{~m}, 1 \mathrm{H}), 7.25-7.50(\mathrm{~m}, 5 \mathrm{H})$; ${ }^{13} \mathrm{C}$ NMR $\left(75 \mathrm{MHz}, \mathrm{CDCl}_{3}\right) \delta 16.5,17.1,29.1,31.1,31.2,44.2,46.1,47.4,63.9,67.1,87.0$, 118.2, 128.5, 129.2, 139.1 ; IR $\left(\mathrm{cm}^{-1}\right)$ 3418, 2840, 2364, 2343, 1743, 1622, 1495, 1265 ; HRMS for $\mathrm{C}_{19} \mathrm{H}_{23} \mathrm{~N}_{2} \mathrm{OClNa}$ calcd. 353.1396; found 353.1397.

6-Deca-1,9-diynyl-1-(2-hydroxy-1-phenyl-ethyl)-piperidine-2-carbonitrile 9c oil, $[\alpha]_{\mathrm{D}}=$ $80\left(\mathrm{c}=1.05, \mathrm{CH}_{2} \mathrm{Cl}_{2}\right) ;{ }^{1} \mathrm{H} \mathrm{NMR}\left(400 \mathrm{MHz}, \mathrm{CDCl}_{3}\right) \delta 1.40-1.70(\mathrm{~m}, 13 \mathrm{H}), 1.82-1.87(\mathrm{~m}, 2 \mathrm{H})$, 1.92- $2.03(\mathrm{~m}, 2 \mathrm{H}), 1.94(\mathrm{t}, J=2.6 \mathrm{~Hz}, 1 \mathrm{H}), 2.21(\mathrm{td}, J=7.0,2.6 \mathrm{~Hz}, 1 \mathrm{H}), 2.30(\mathrm{td}, J=7.0$, $2.1 \mathrm{~Hz}, 1 \mathrm{H}), 3.57(\mathrm{dd}, J=4.9,2.1 \mathrm{~Hz}, 1 \mathrm{H}), 3.86(\mathrm{ddd}, J=11.3,3.6,3.4 \mathrm{~Hz}, 1 \mathrm{H}), 3.97(\mathrm{dd}, J=$ 5.0, 3.4 Hz, 1H), $4.05(\mathrm{dt}, J=11.4,5.4 \mathrm{~Hz}, 1 \mathrm{H}), 4.16(\mathrm{~m}, 1 \mathrm{H}), 7.25-7.50(\mathrm{~m}, 5 \mathrm{H}) ;{ }^{13} \mathrm{C} \mathrm{NMR}$ $\left(75 \mathrm{MHz}, \mathrm{CDCl}_{3}\right) \delta 17.0,18.4,18.8,28.3,28.5,29.2,31.2,46.1,47.4,63.1,67.0,68.3,76.0$, 84.8, 89.0, 118.5, 128.4, 128.5, 129.1, 139.2 ; IR $\left(\mathrm{cm}^{-1}\right)$ 3478, 3290, 2934, 2858, 2361, 2226, 2115, 1749, 1653, 1494; HRMS for $\mathrm{C}_{24} \mathrm{H}_{30} \mathrm{~N}_{2} \mathrm{ONa}$ calcd. 385.2256; found 385.2256. 
S3
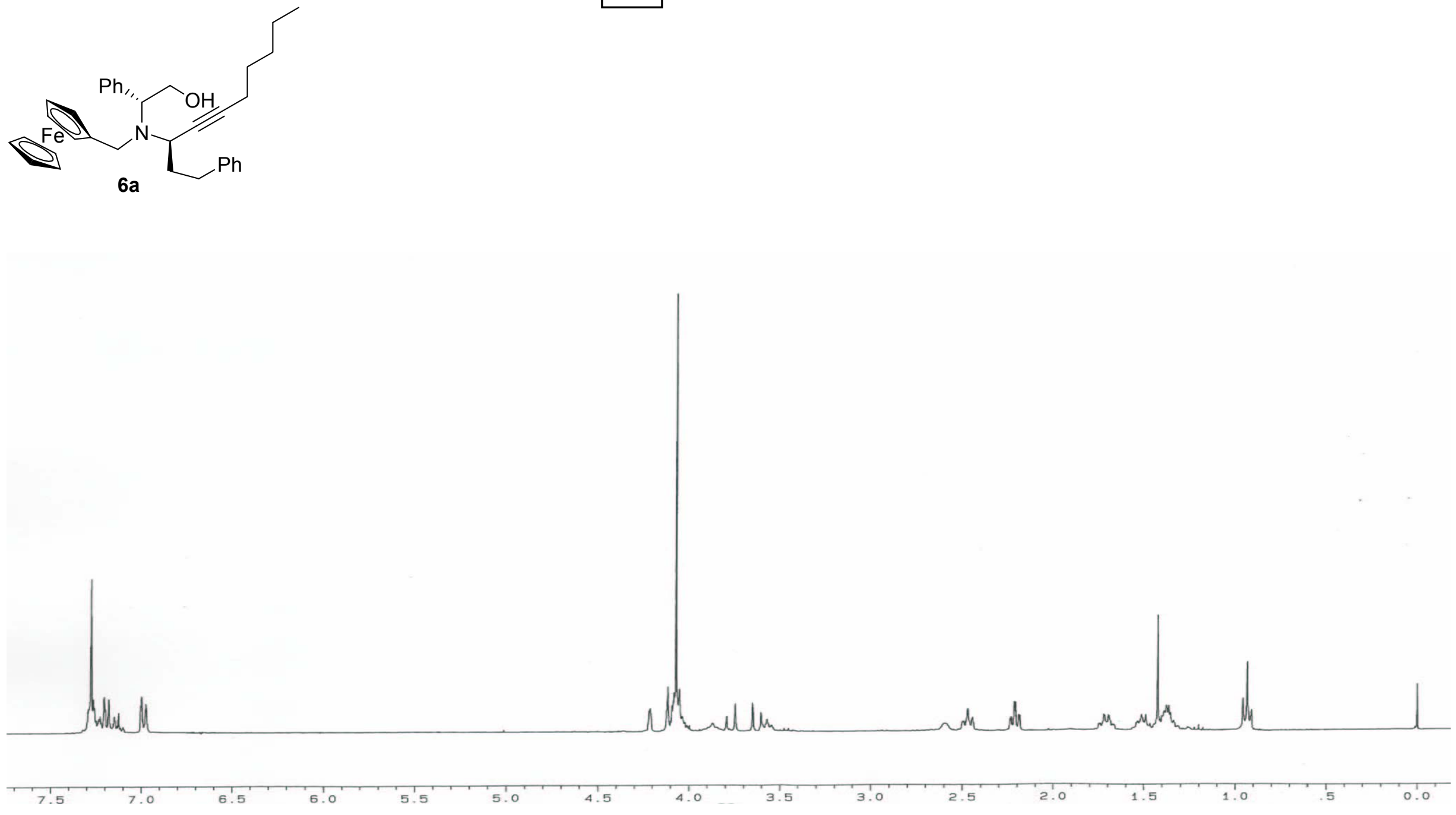


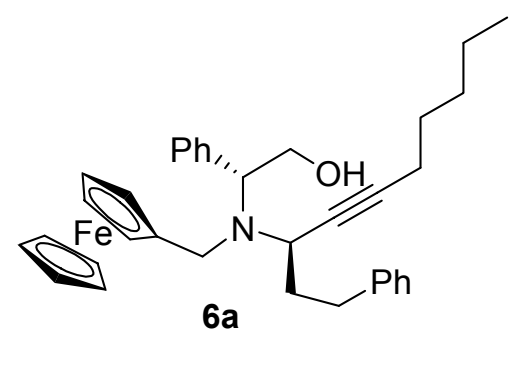

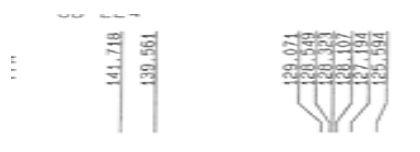

54

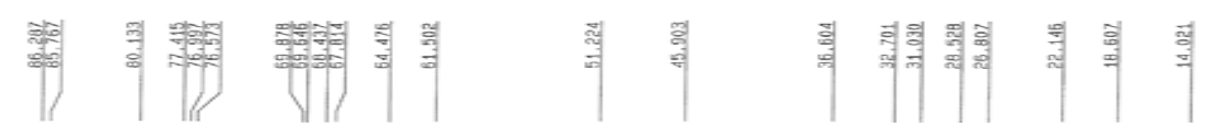

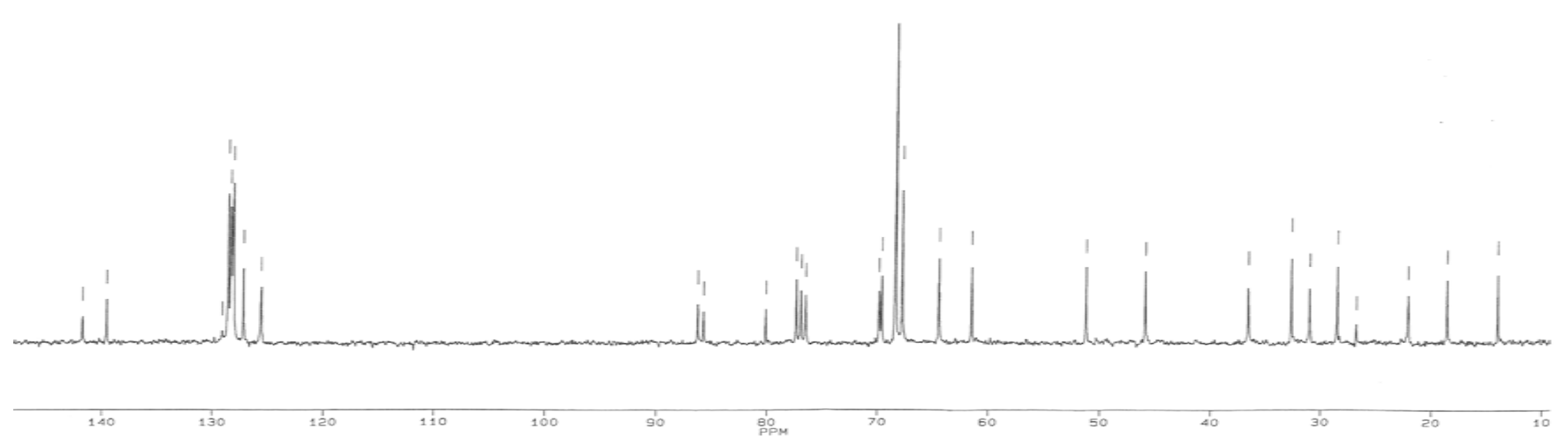




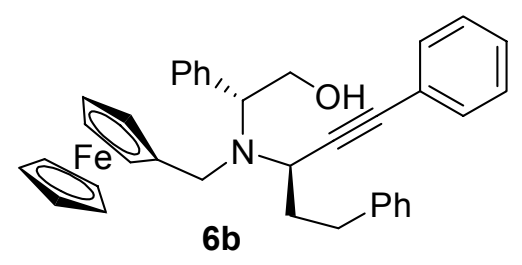

S5
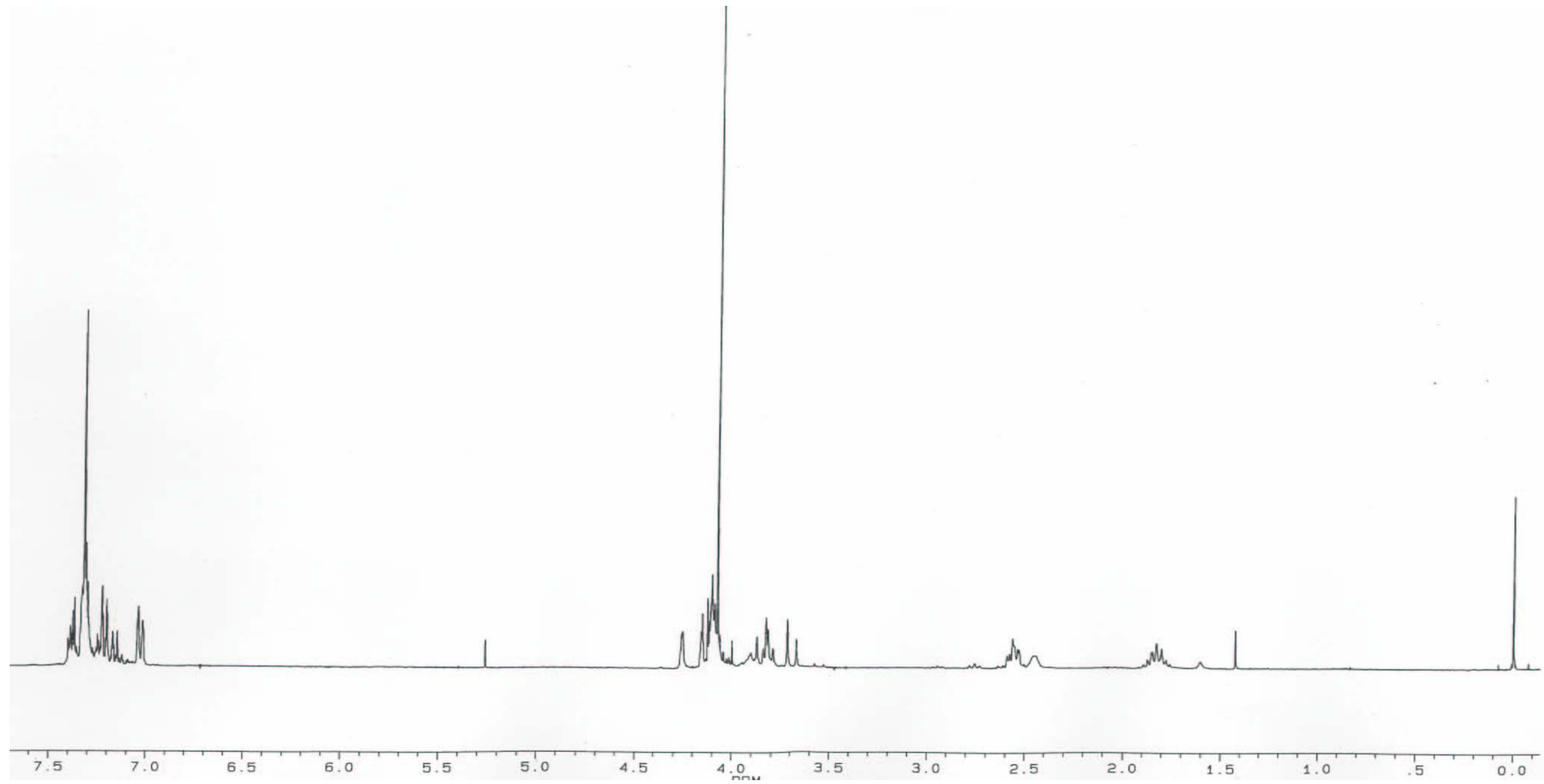


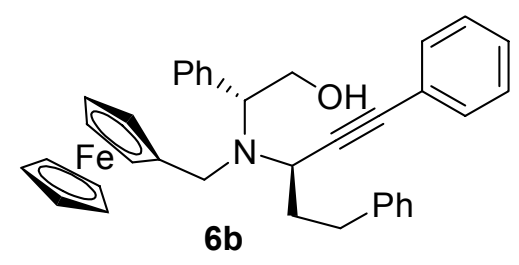

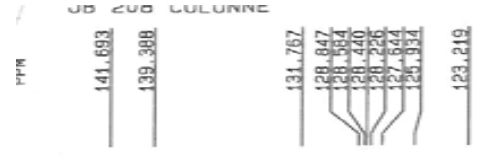

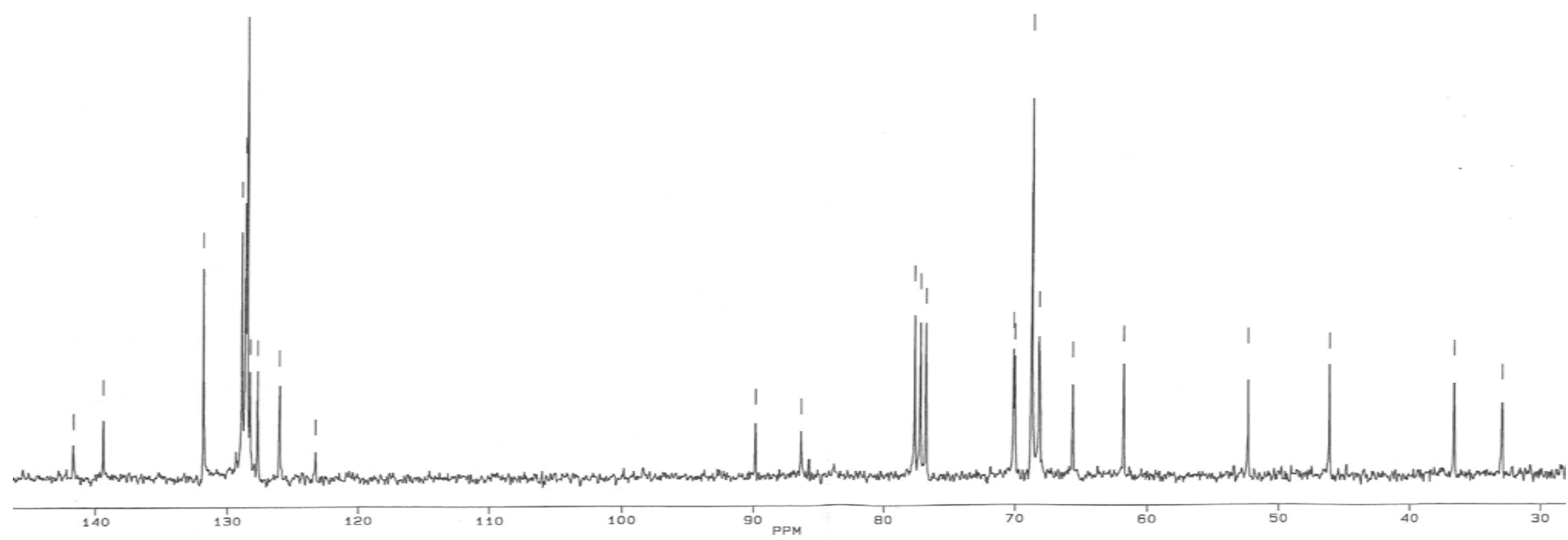



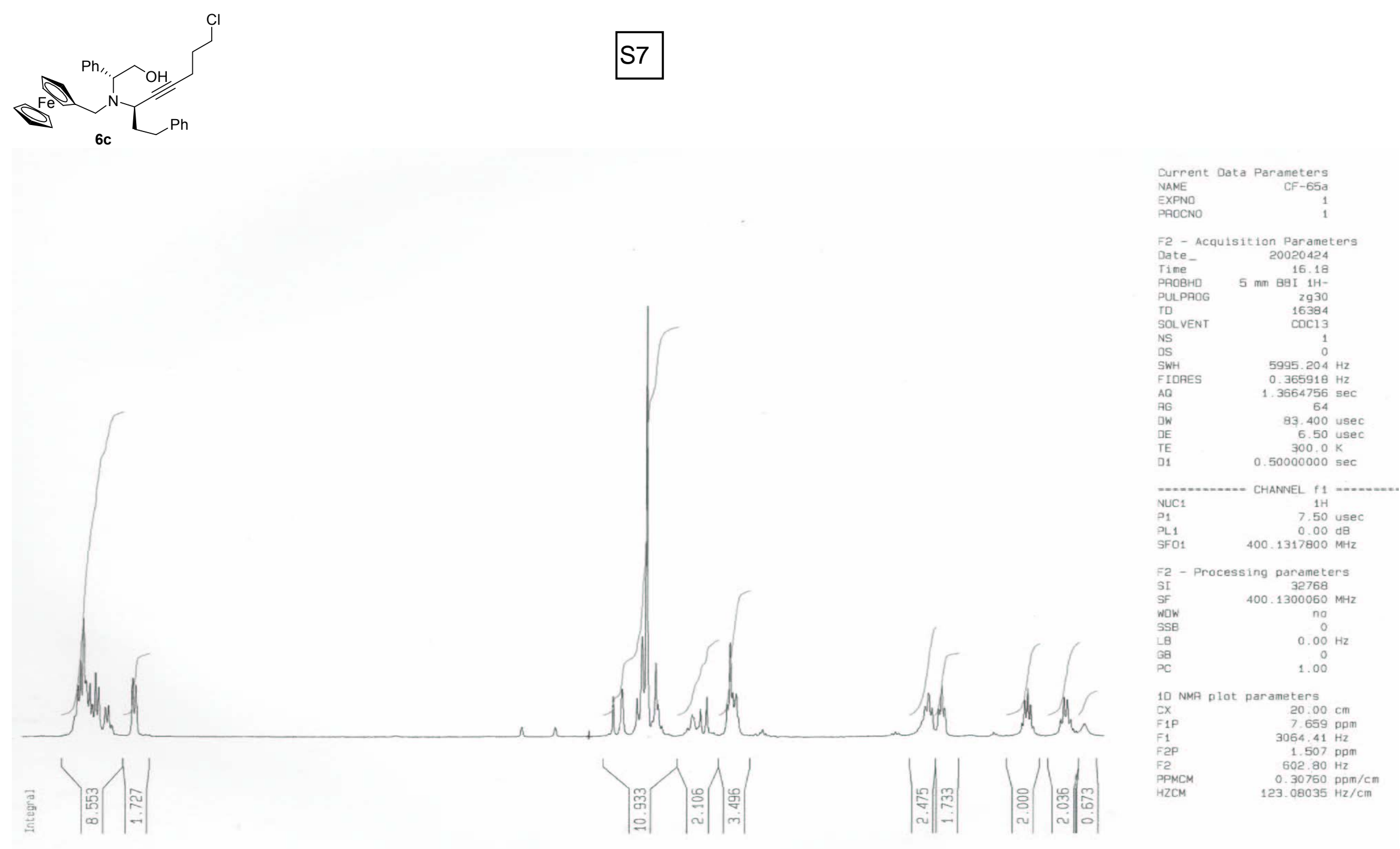


$$
\text { (c) }
$$

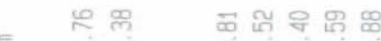

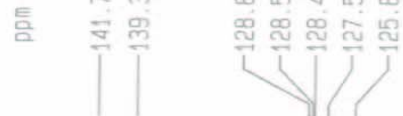

$$
\text { it }
$$

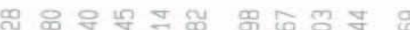

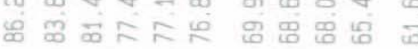

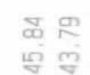

ले ले लै

cf65a

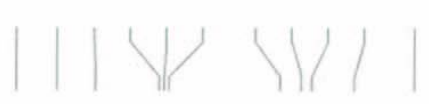

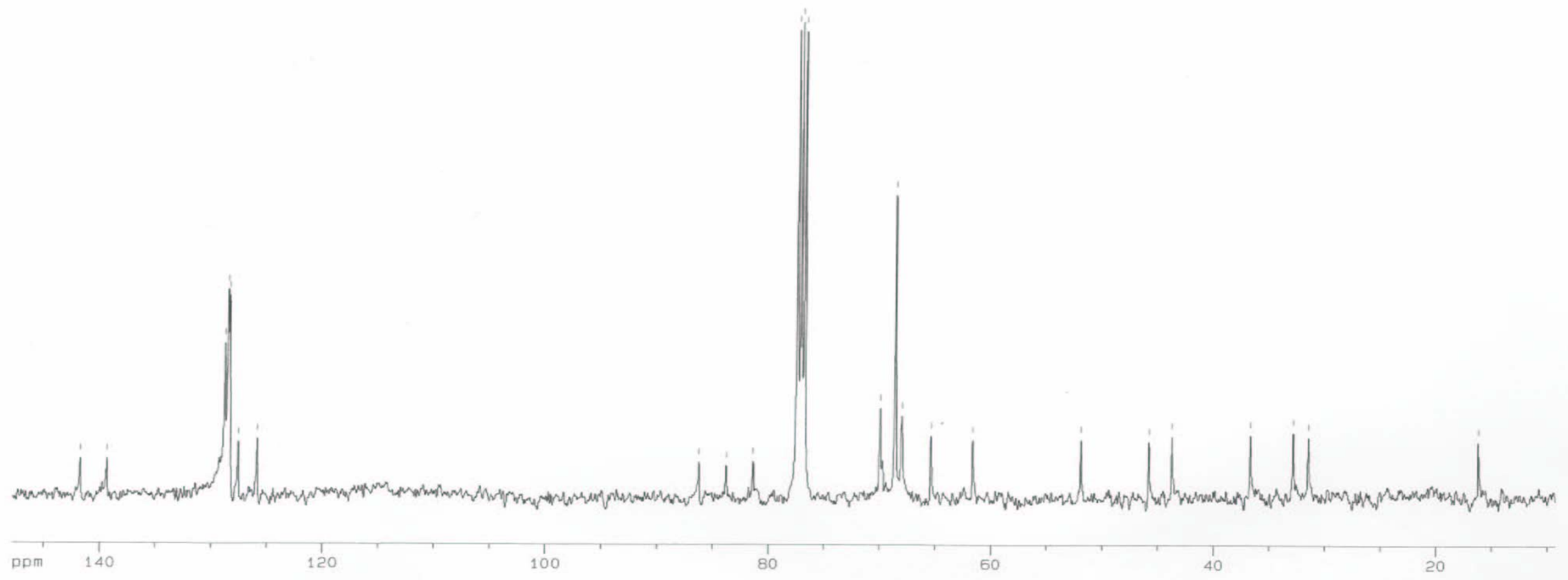



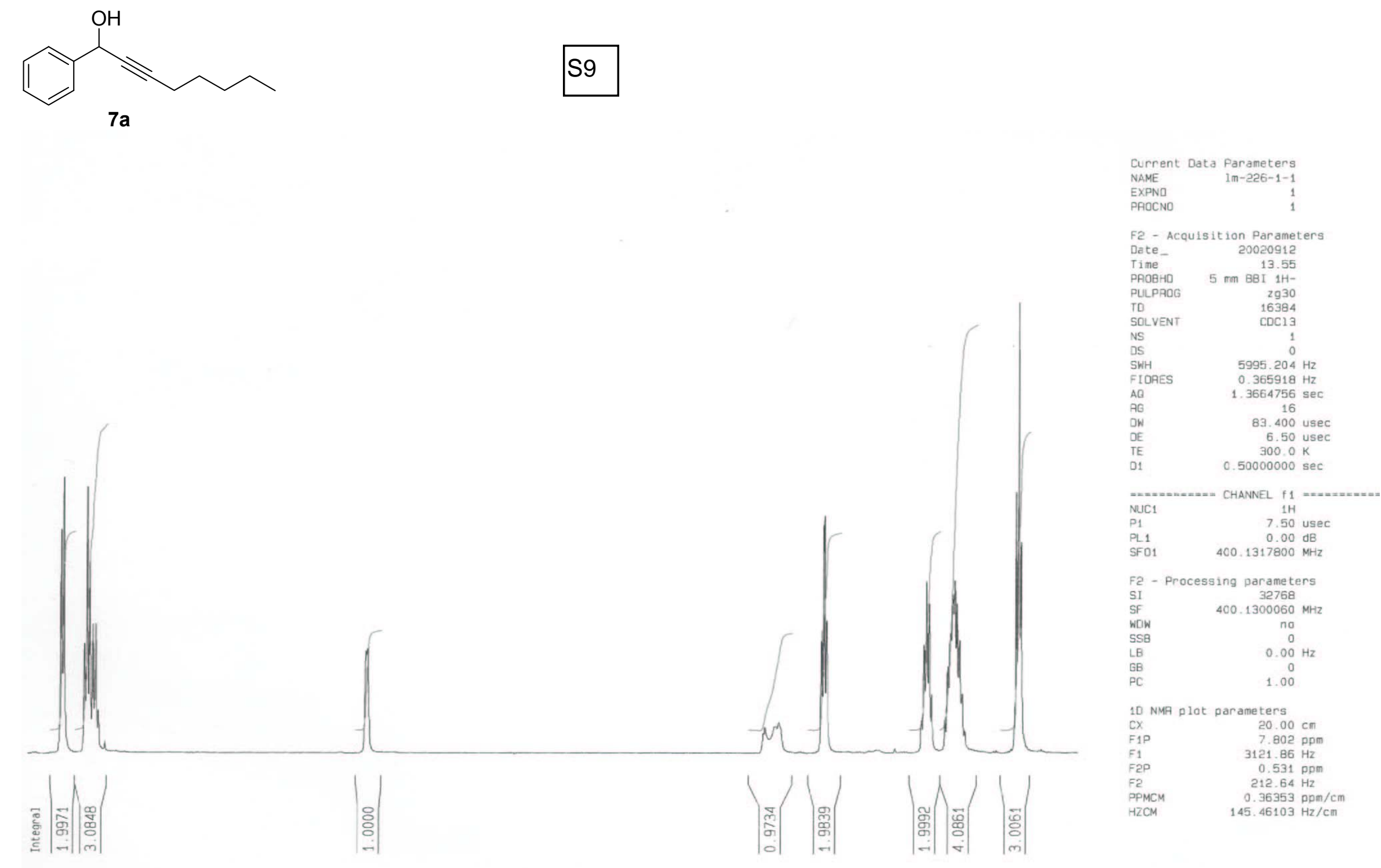


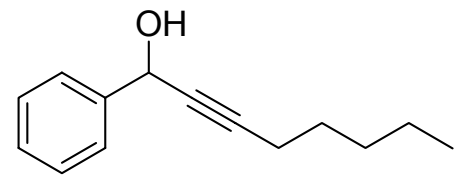

$7 a$
$S 10$

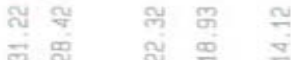

Im 226
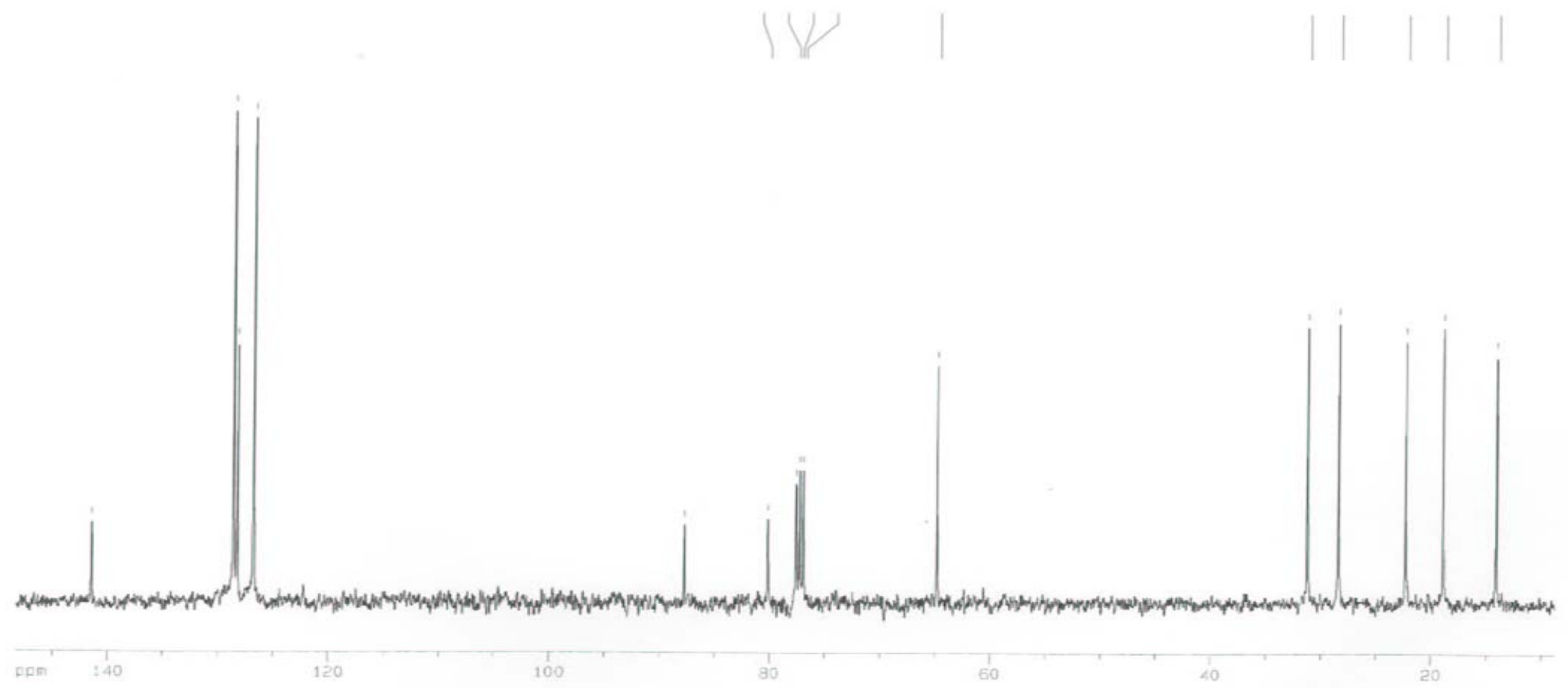

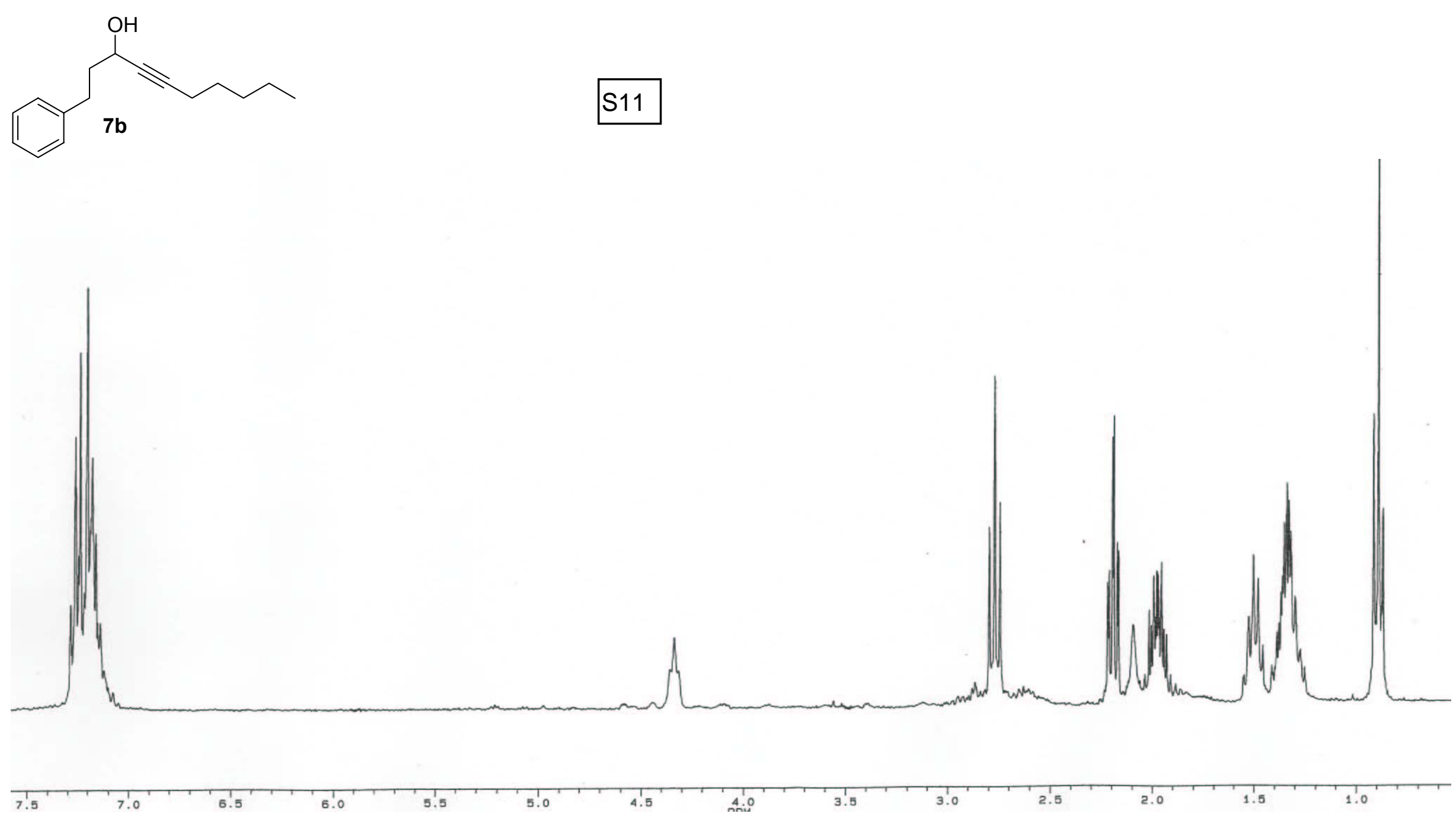

5.0

4.5 4.0 3.5 $3.0^{\circ}$ 2.5 2.0 ${ }_{1.5}^{1}$ $1.0^{\prime}$ 


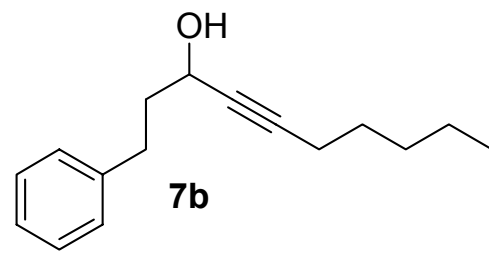

S12

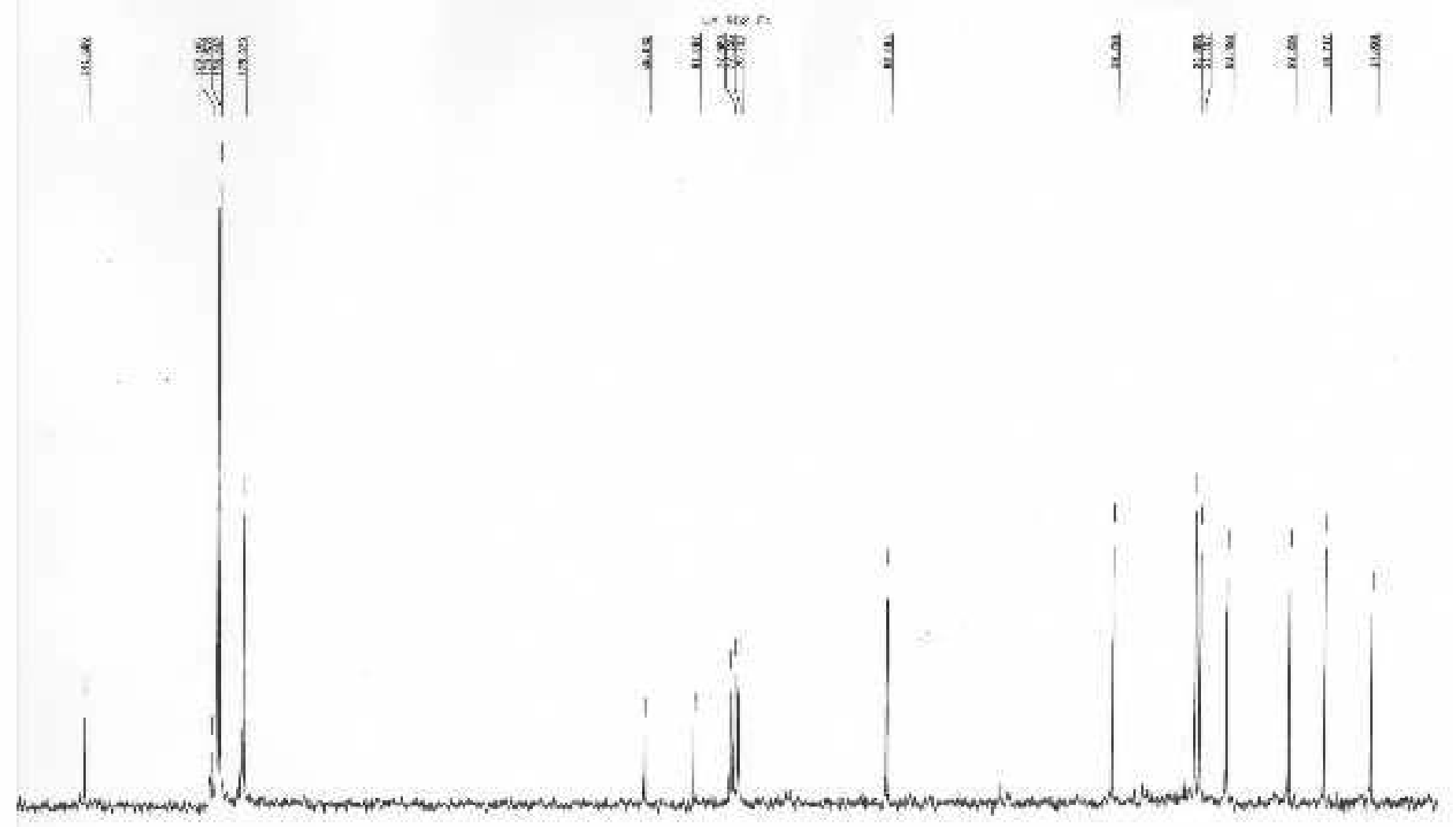

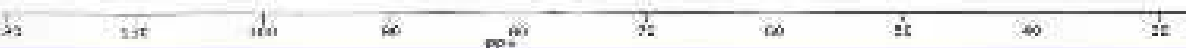



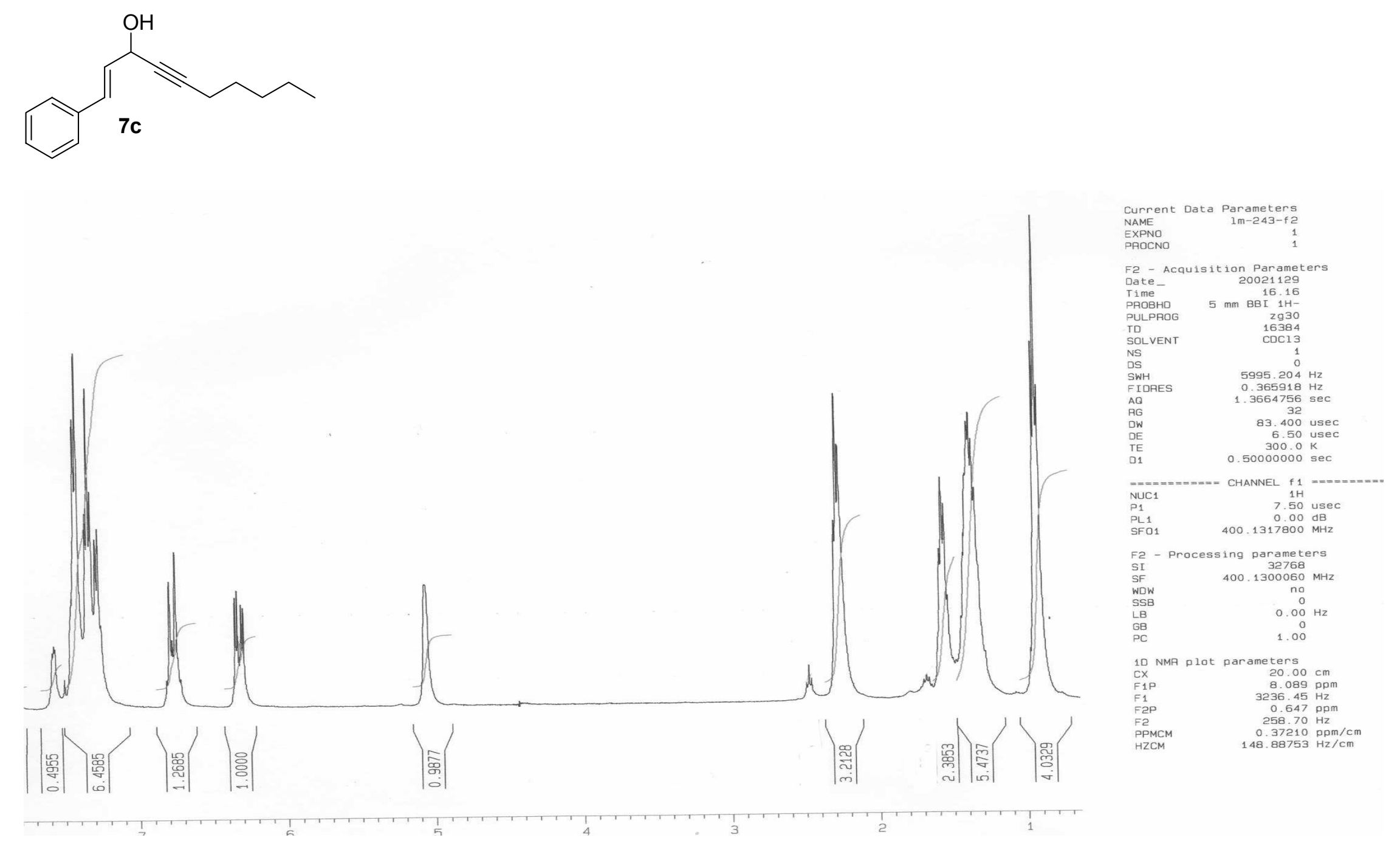


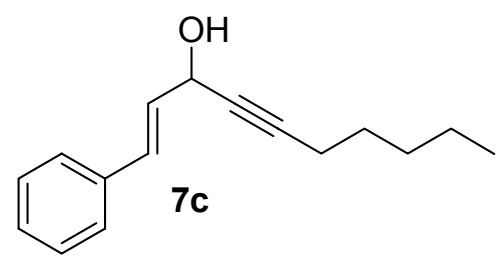

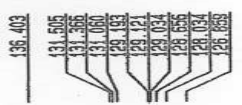

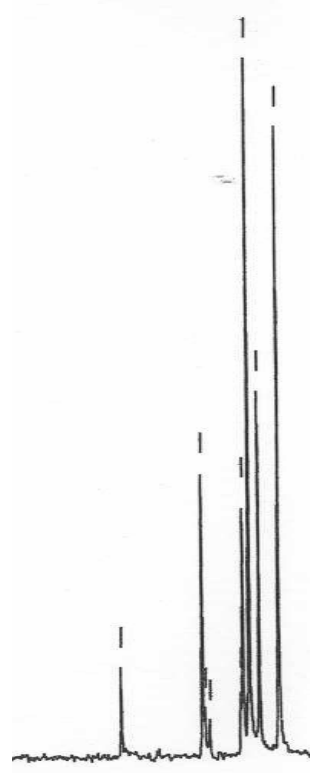

140

.
S14

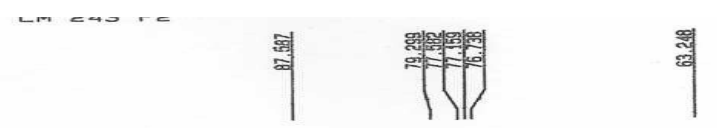

非帏是 

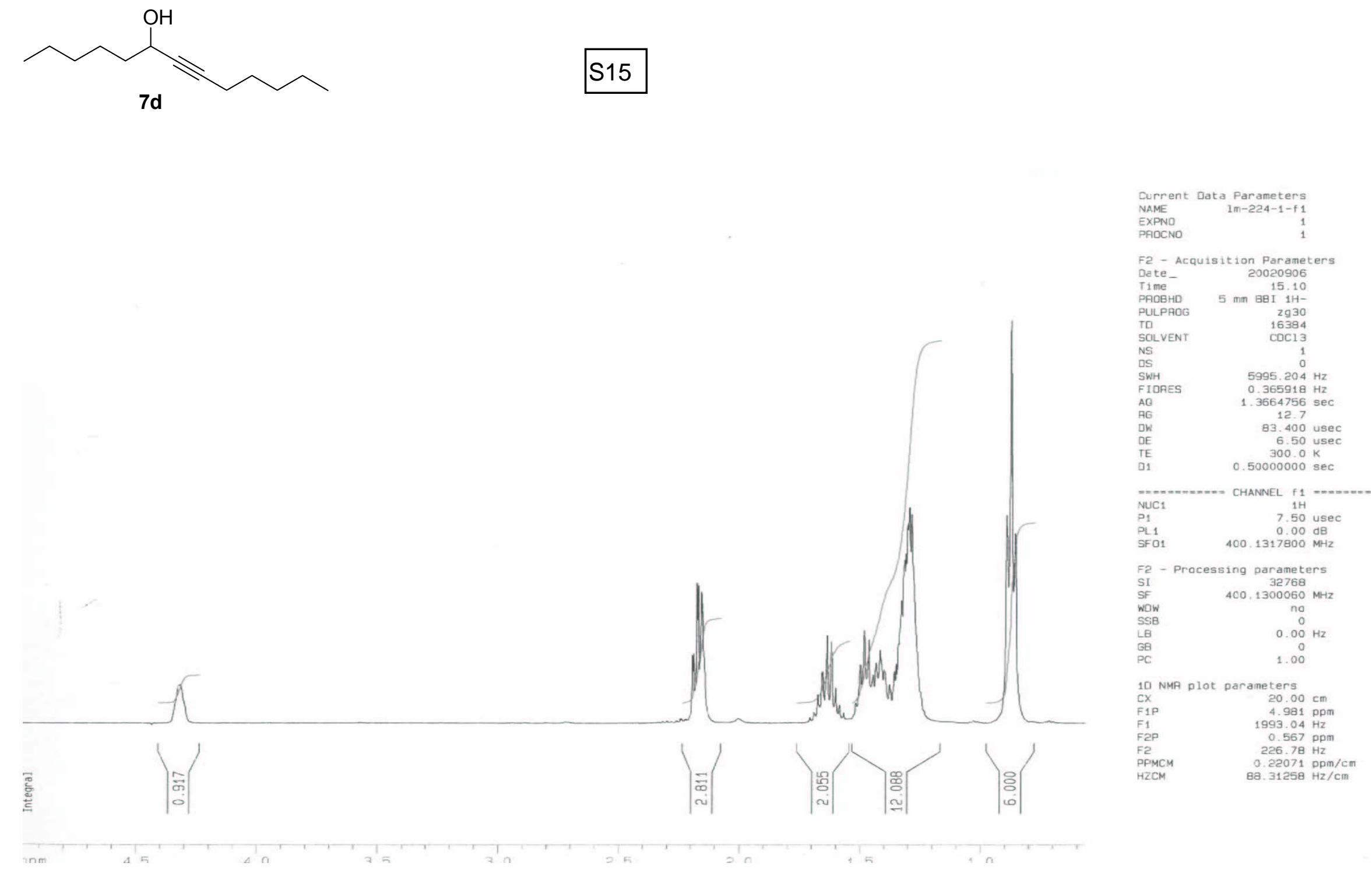


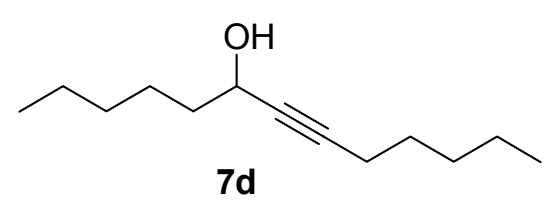

S16

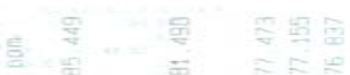

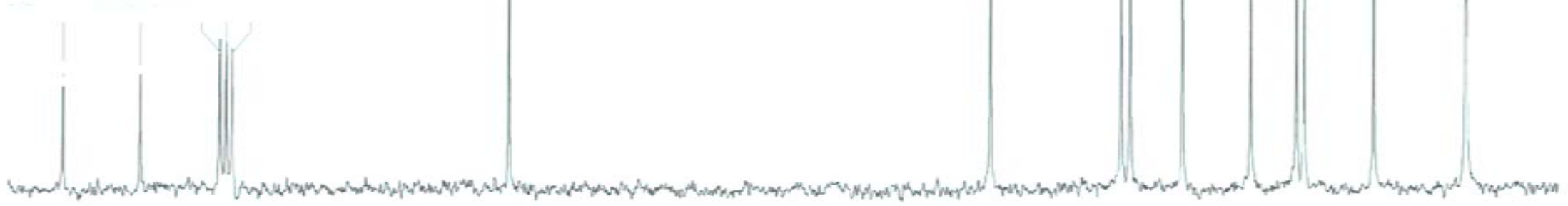




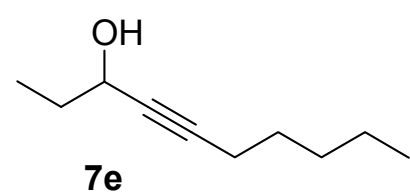

$$
\text { S17 }
$$
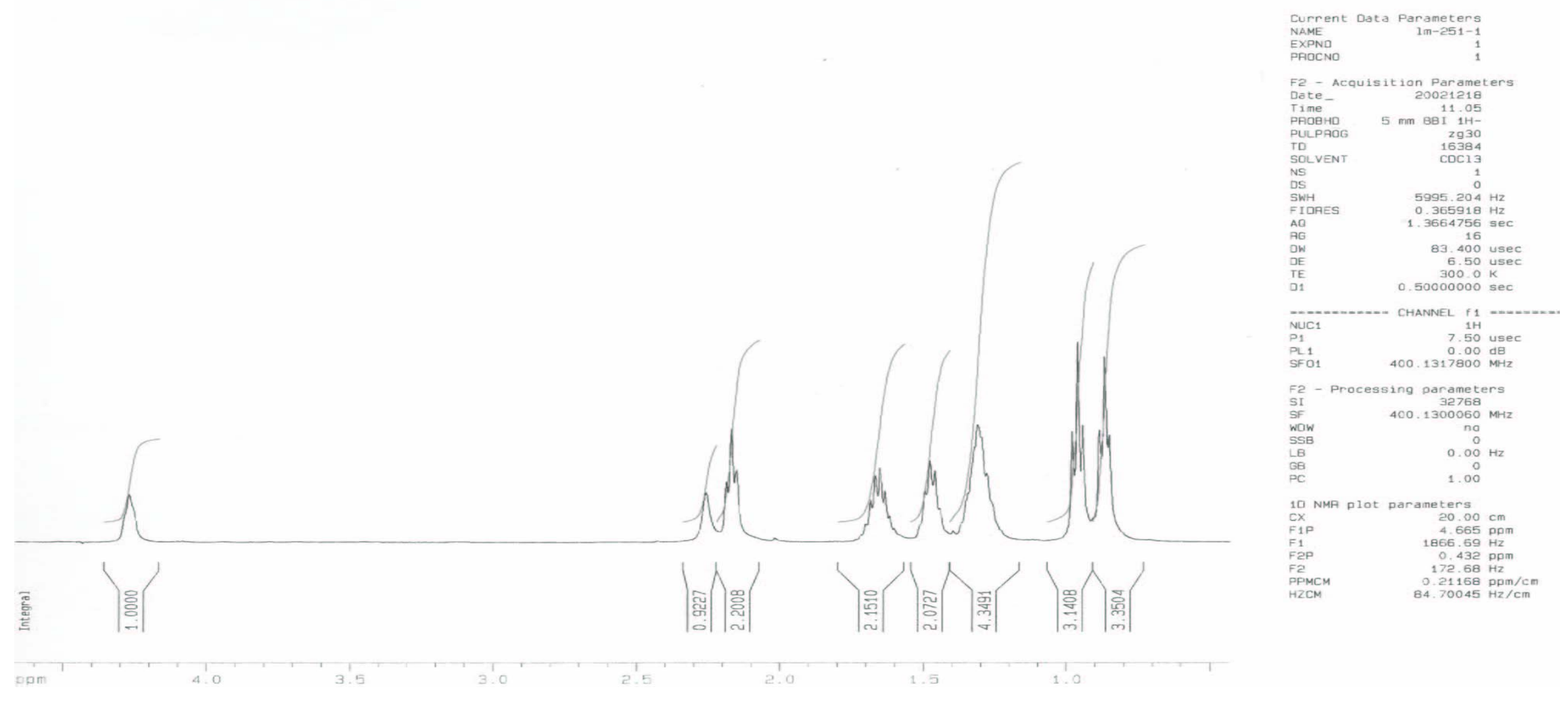


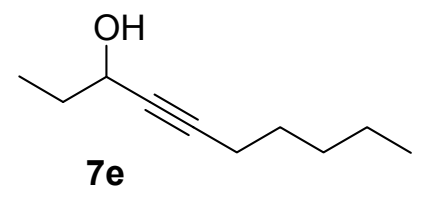

$$
\text { S18 }
$$

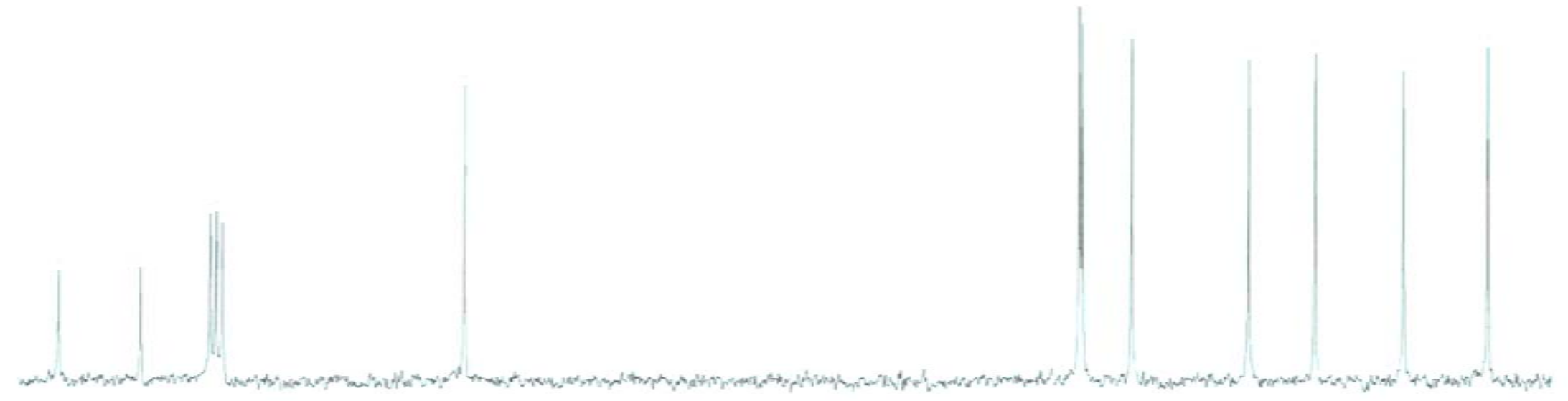




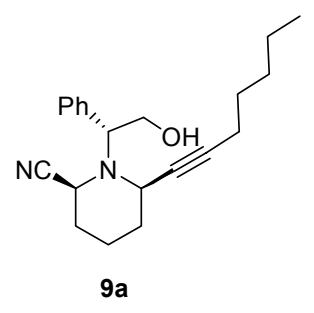

$9 a$
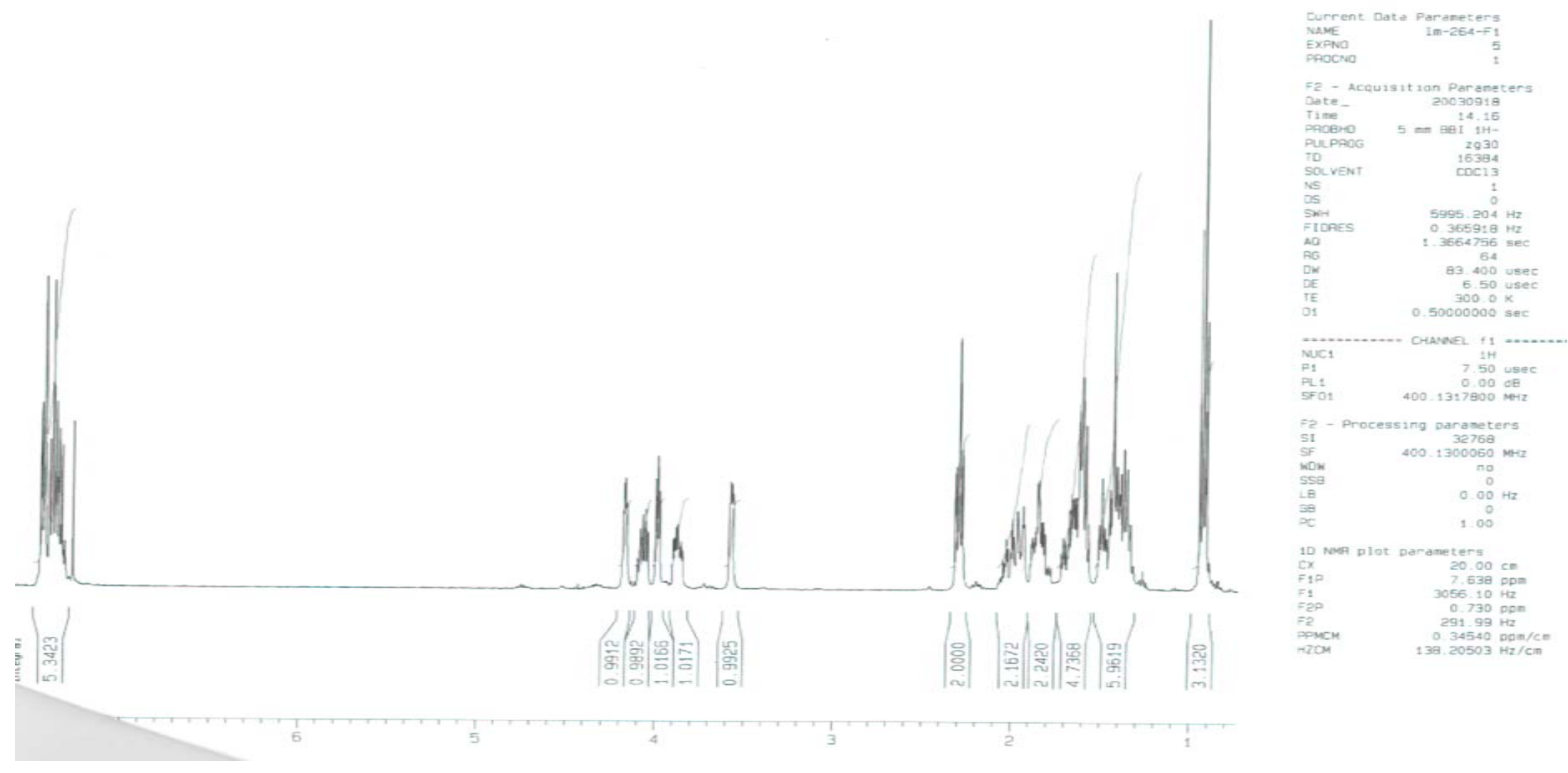


$$
\text { (1) }
$$

9a

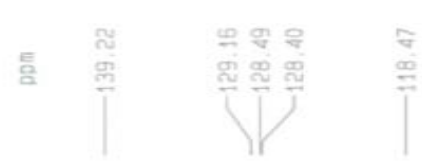

इำ 을 के ज्ञ

1m $264 \mathrm{~F} 1$
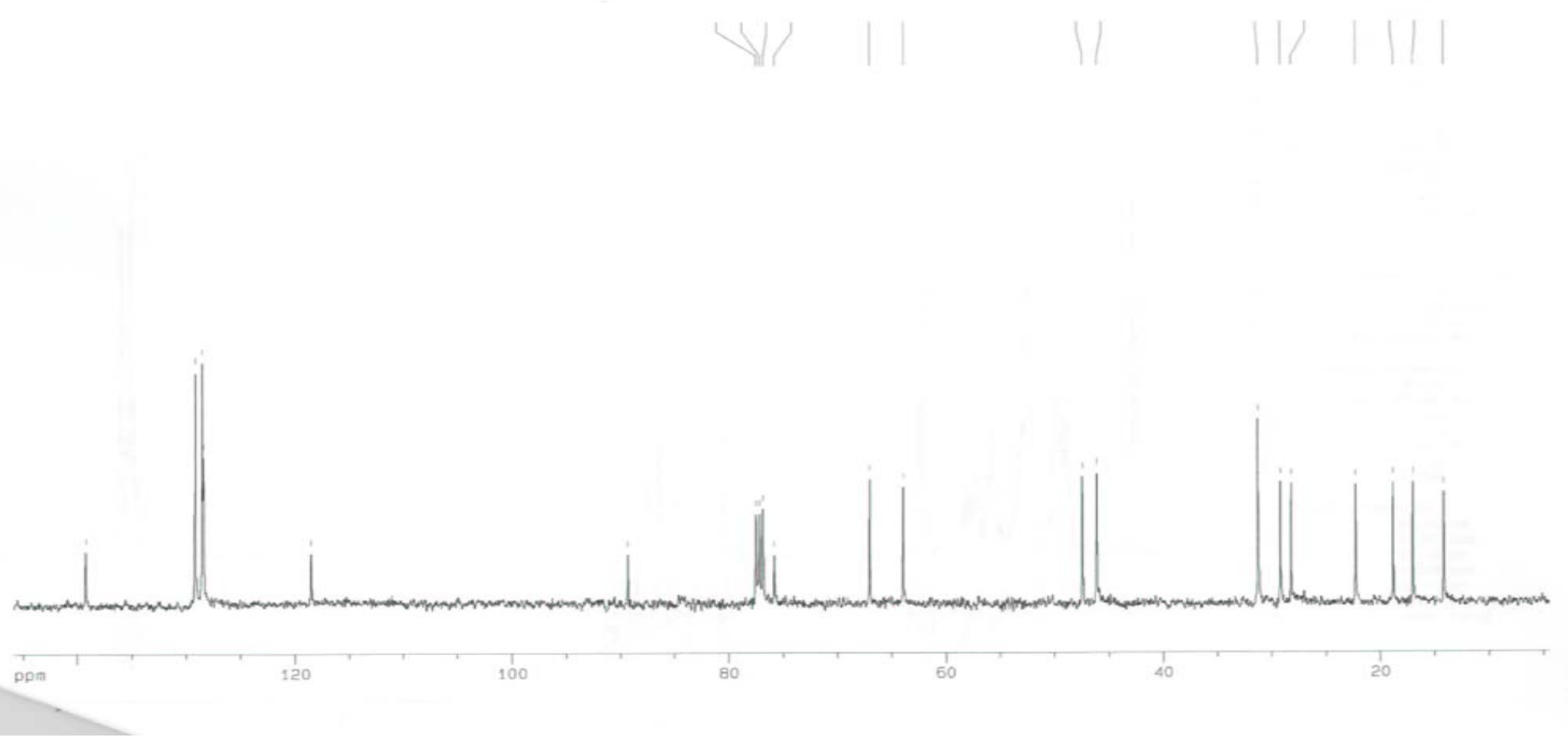


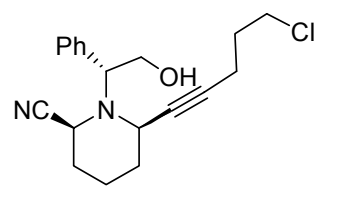

$9 b$

$$
\mathrm{S} 21
$$

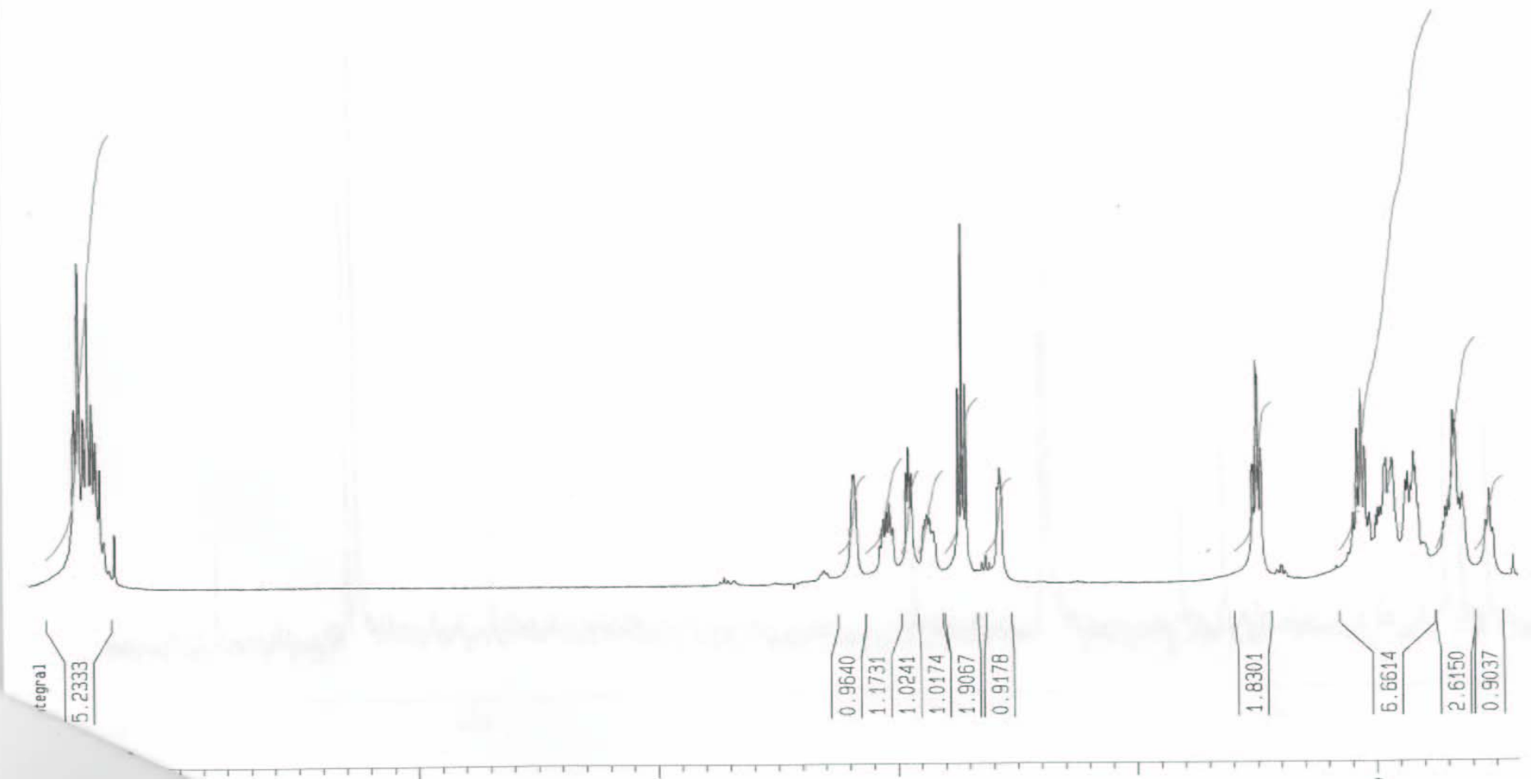

Current Data Parameters
NAME

NAME

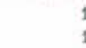

F2 - Acquisition Parameters

Date-

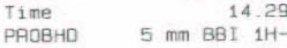

PULPAOG Z 2930

To

2930
16384
$\operatorname{CDC} 13$
1

$\begin{array}{ll}\text { NS } & 1 \\ \text { DS } & 0\end{array}$

$\begin{array}{ll}\text { SWH } & 5995.204 \mathrm{~Hz} \\ \text { FIORES } & 0.365918 \mathrm{~Hz}\end{array}$

AQ $\quad 1.3664756 \mathrm{sec}$

$\begin{array}{lr}\text { DW } & 83.400 \text { usec } \\ \text { DE } & 6.50 \text { usec }\end{array}$

0.50000000 sec

NUC1

$\begin{array}{lr}\text { P1 } & 7.50 \text { use } \\ \text { PL1 } & 0.00 \mathrm{~dB}\end{array}$

Fe - Processing parameters

$\begin{array}{ll}\text { FE - Processing parameters } & \text { 3768 } \\ \text { SI } & \\ \text { SF } & 400,1300060 \mathrm{MHZ}\end{array}$

WOW

LB
GB
PC

1.00

10 NMA plot parameters

$\begin{array}{ll}\mathrm{CX} & 20.00 \mathrm{~cm} \\ \mathrm{~F} 1 \mathrm{P} & 7.634 \mathrm{pm}\end{array}$

$\begin{array}{lr}\mathrm{F} 1 & 3054.78 \mathrm{~Hz} \\ \mathrm{~F} 2 \mathrm{P} & 1.40 \mathrm{ppm}\end{array}$

$\begin{array}{ll}F 2 & 563.55 \mathrm{~Hz} \\ \text { PPMCM } & 0.31130 \mathrm{pm} / \mathrm{Cm}\end{array}$

$\begin{array}{ll}\text { HZCM } & 0.31130 \mathrm{ppm} / \mathrm{cm} \\ 124.56156 \mathrm{~Hz} / \mathrm{cm}\end{array}$ 
Cl

$9 b$

S22

言告

罗乐

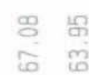

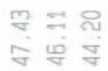

뜩 용

용 뭉

$1 m-277-f 1$

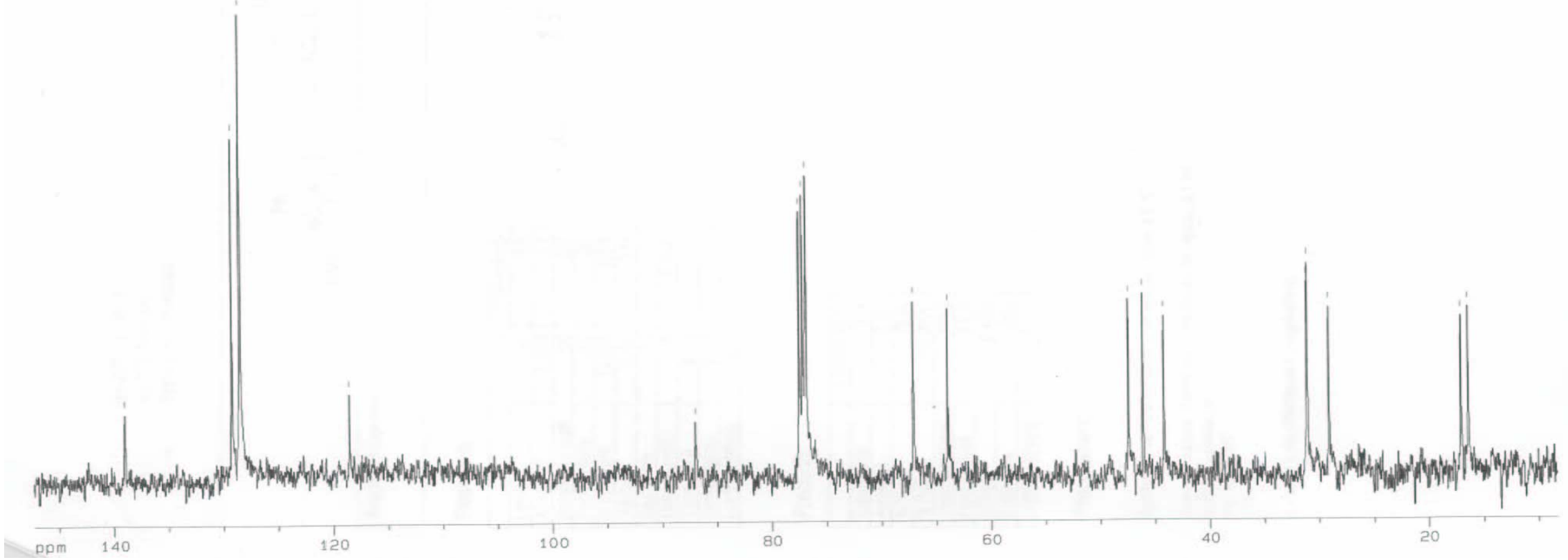



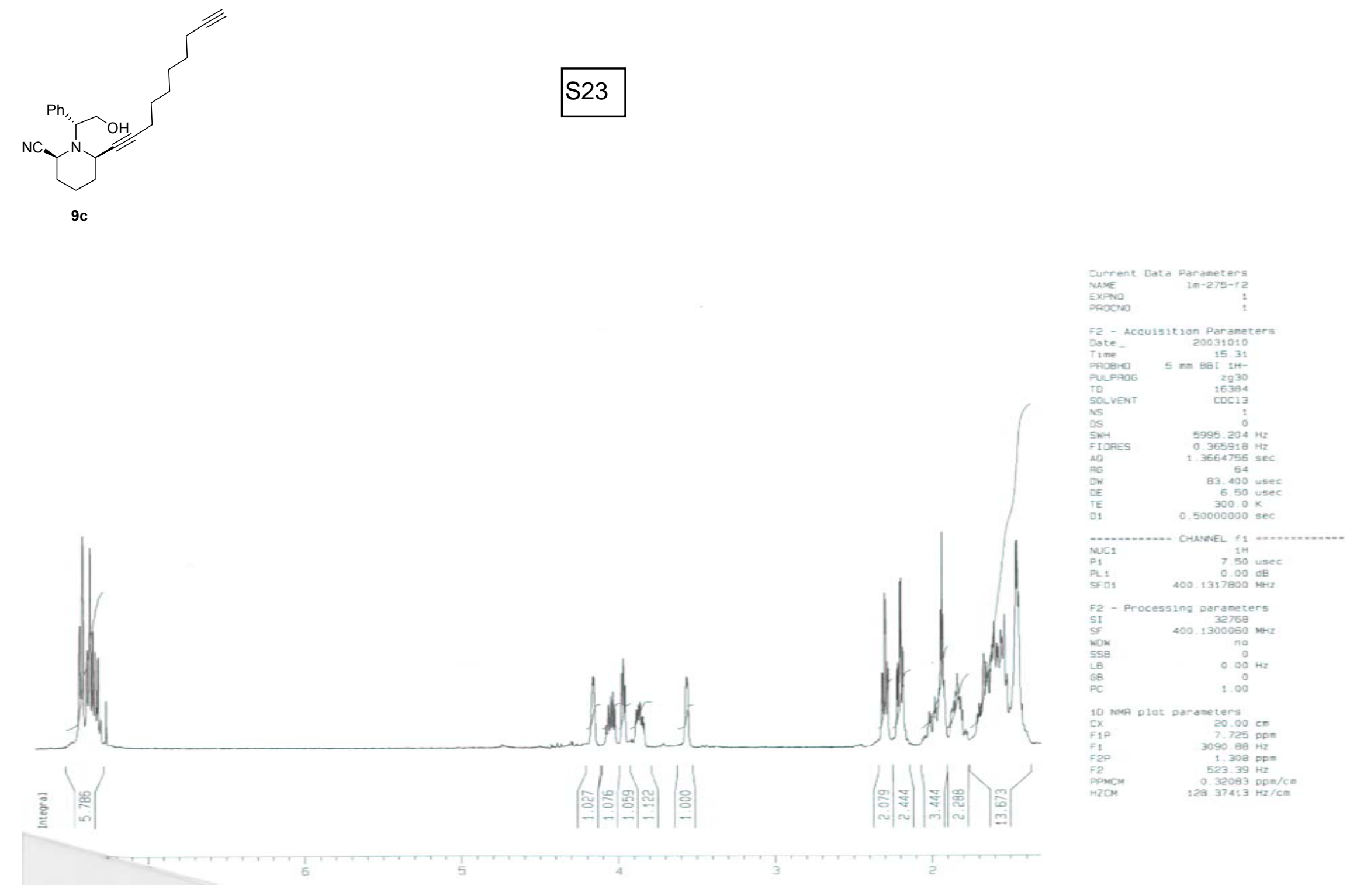


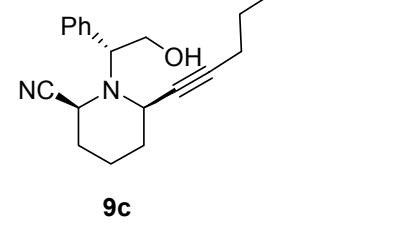

$9 c$

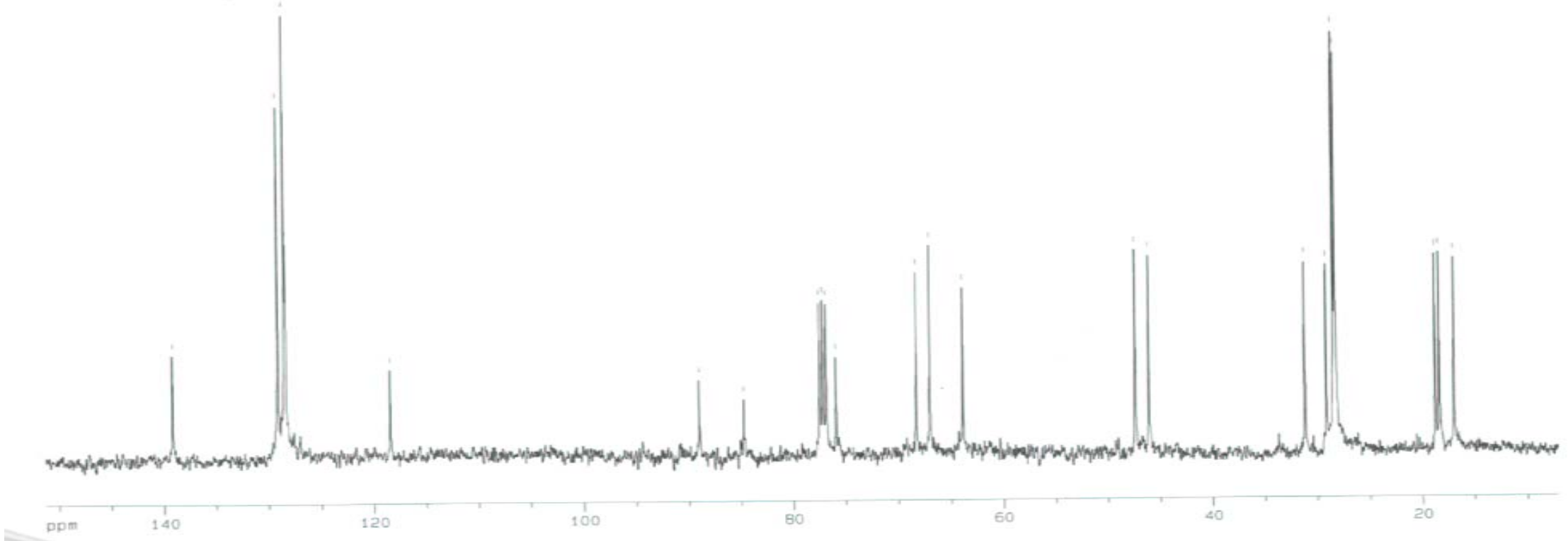

